\title{
Rapid automatic assessment of microvascular density in sidestream dark field images
}

\author{
Rick Bezemer $\cdot$ Johannes G. Dobbe $\cdot$ Sebastiaan A. Bartels $\cdot$ E. Christiaan Boerma \\ Paul W. G. Elbers $\cdot$ Michal Heger $\cdot$ Can Ince
}

Received: 30 March 2011/ Accepted: 4 August 2011/Published online: 31 August 2011

(C) International Federation for Medical and Biological Engineering 2011

\begin{abstract}
The purpose of this study was to develop a rapid and fully automatic method for the assessment of microvascular density and perfusion in sidestream dark field (SDF) images. We modified algorithms previously developed by our group for microvascular density assessment and introduced a new method for microvascular perfusion assessment. To validate the new algorithm for microvascular density assessment, we reanalyzed a selection of SDF video clips $(n=325)$ from a study in intensive care patients and compared the results to (semi-)manually found microvascular densities. The method for microvascular perfusion assessment (temporal SDF image contrast analysis, tSICA) was tested in several video simulations and in one high quality SDF video clip where the
\end{abstract}

R. Bezemer $(\bowtie) \cdot$ S. A. Bartels · E. Christiaan Boerma .

C. Ince

Department of Translational Physiology, Academic Medical

Center, University of Amsterdam, Meibergdreef 9, 1105,

AZ, Amsterdam, The Netherlands

e-mail: r.bezemer@amc.uva.nl

R. Bezemer · S. A. Bartels · C. Ince

Department of Intensive Care, Erasmus University Hospital,

Rotterdam, The Netherlands

J. G. Dobbe

Department of Biomedical Engineering and Physics, Academic

Medical Center, University of Amsterdam, Amsterdam,

The Netherlands

E. Christiaan Boerma · P. W. G. Elbers

Department of Intensive Care and Department of Cardiac

Anesthesia, Medical Center Leeuwarden, Leeuwarden,

The Netherlands

M. Heger

Department of Experimental Surgery, Academic Medical Center, University of Amsterdam, Amsterdam, The Netherlands microcirculation was imaged before and during circulatory arrest in a cardiac surgery patient. We found that the new method for microvascular density assessment was very rapid ( $<30 \mathrm{~s} / \mathrm{clip})$ and correlated excellently with (semi-) manually measured microvascular density. The new method for microvascular perfusion assessment (tSICA) was shown to be limited by high cell densities and velocities, which severely impedes the applicability of this method in real SDF images. Hence, here we present a validated method for rapid and fully automatic assessment of microvascular density in SDF images. The new method was shown to be much faster than the conventional (semi-) manual method. Due to current SDF imaging hardware limitations, we were not able to automatically detect microvascular perfusion.

Keywords SDF imaging - Laser speckle imaging . Image contrast analysis - TSICA · Microcirculation . Microvascular density $\cdot$ Microvascular perfusion . Videomicroscopy

\section{Introduction}

Orthogonal polarization spectral (OPS) imaging [17] and sidestream dark field (SDF) imaging are microscopic techniques incorporated in hand held microscopes that allow the assessment of microvascular density and perfusion in various clinical settings at the bed side [9, 10]. Using sublingual SDF imaging it has been revealed that microcirculatory alterations are key in the development of (multiple) organ failure in critically ill patients, especially in sepsis and shock [18]. Normalizing the microcirculation has therefore become the focus of new clinical trials and therapeutic strategies and consequently, microcirculatory 
imaging is gaining a more prominent role in clinical research $[4,11]$. For evaluation of the effects of interventions and (drug) therapy, SDF images are analyzed to assess (alterations in) microvascular density and perfusion [5, 9-11, 22, 23].

To reduce the time required for SDF image analysis of microvascular density and perfusion, Dobbe et al. [13] have developed a method that has been commercialized into a software package called Automated Vascular Analysis (AVA). This method automatically determines vessel center lines in straight and curved vessel segments, which are validated as being actual vessels based on their automatically assigned focus score. AVA has been validated using video simulations of vessels with known lengths, diameters, and RBC velocities [13] and has been used in several clinical studies (e.g., 13, 16). While the method was successful in detecting vessel center lines in video simulations, in real SDF video clips this was shown to be more difficult in case of suboptimal image focus (vessel validation is based on focus). Therefore, the user is allowed to manually add falsely excluded vessels and delete falsely included vessels. When all vessel segments are identified, microcirculatory density can be quantified and red blood cell (RBC) velocities can be determined in individual vessels using space-time diagrams. However, detection of RBC velocities is limited by the SDF imaging hardware where a relatively low imaging rate of $25 \mathrm{~Hz}$ compared to the RBC velocities causes blurring of RBC patterns within vessels. To address the latter, semi-quantitative scoring methods have been developed to characterize microcirculatory flow as 'no flow', 'intermittent flow', 'sluggish flow', and 'continuous flow' [4]. Manually assigning a flow score to each detected vessel center line allows the calculation of total and perfused vessel density (TVD and PVD, respectively) and the portion of perfused vessels (PPV) [11].

The semi-automated assessment of TVD, PVD, and PPV using the AVA software is a time consuming venture (10-30 min, depending on the level of experience of the user) and requires a significant amount of user interaction. Therefore, the primary aim of this study was to improve the microvascular density assessment to allow rapid $(<30 \mathrm{~s})$ and fully automatic (no user input) determination of the TVD, by modifying the vessel recognition/validation algorithm that is incorporated in the AVA software. The secondary aim was to develop a binary perfusion detection method for SDF images based on temporal pixel intensity fluctuations, which we termed temporal SDF image contrast analysis (tSICA). To validate the algorithm for rapid and fully automatic detection of TVD, we reanalyzed a selection of SDF video clips that were already analyzed using AVA software for a study published elsewhere [5] and compared the results to (semi-)manually found microvascular densities. Subsequently, we tested the performance of the tSICA method for the binary detection of perfusion in several video simulations similar to those used previously [13] and characterized the effects of cell velocity, cell density, and the SDF imaging rate on the ability of tSICA to detect perfusion. Finally, the use of tSICA was demonstrated in one high quality (in terms of focus and contrast) SDF video clip where the microcirculation was imaged before and during circulatory arrest in cardiac surgery patients [15].

\section{Materials and methods}

\subsection{SDF imaging}

The sublingual microcirculation was monitored using an SDF imaging device (Microvision Medical BV, Amsterdam, the Netherlands) $[13,15]$. A detailed description of the SDF imaging technology is provided elsewhere [13, 16]. An imaging objective with a $5 \times$ optical magnification resulted in microcirculatory images with a resolution of $1.4 \times 1.5 \mu \mathrm{m} /$ pixel [13]. Microcirculatory images were acquired at a rate of $25 \mathrm{~Hz}$ with a size of $720 \times 576$ pixels, corresponding to a field of view of $1.08 \times 0.81 \mathrm{~mm}$. The images were stored on DVI tape and saved onto a computer in DV-AVI file format for further offline analysis. The SDF images were analyzed using AVA software (Microvision Medical BV, Amsterdam, the Netherlands), which has been developed by our group and described in detail elsewhere [13]. Microvascular density is calculated as the microvascular length $[\mathrm{mm}]$ divided by the imaged tissue area $\left[\mathrm{mm}^{2}\right]$. The microcirculation was scored for total vessel density (TVD in $\mathrm{mm}$ vessel $/ \mathrm{mm}^{2}$ imaged area $\left[\mathrm{mm} / \mathrm{mm}^{2}\right]$ ), perfused vessel density (PVD $\left[\mathrm{mm} / \mathrm{mm}^{2}\right]$ ) [11], the portion of perfused vessels $(\mathrm{PPV}=(\mathrm{PVD} / \mathrm{TVD}) \times 100 \quad[\%])$, and microcirculatory flow index (MFI [AU]) [4], in accordance to the guidelines for microcirculatory image acquisition and analysis [11].

\subsection{Microvascular density assessment}

The new method for rapid and fully automatic detection of TVD is based on the abovementioned AVA software [13]. A diagram schematically presenting the analysis steps required for microvascular density and perfusion assessment is depicted in Fig. 1. First, a specified image sequence was stabilized using 2D cross correlation and time-averaged to fill up RBC interruptions caused by plasma gaps and white blood cells. For microvascular density assessment, the number of images per sequence ranged from 5 to 25 images, depending on image stability; i.e., within an image sequence, the maximum allowed translation was 


\section{Image sequence}

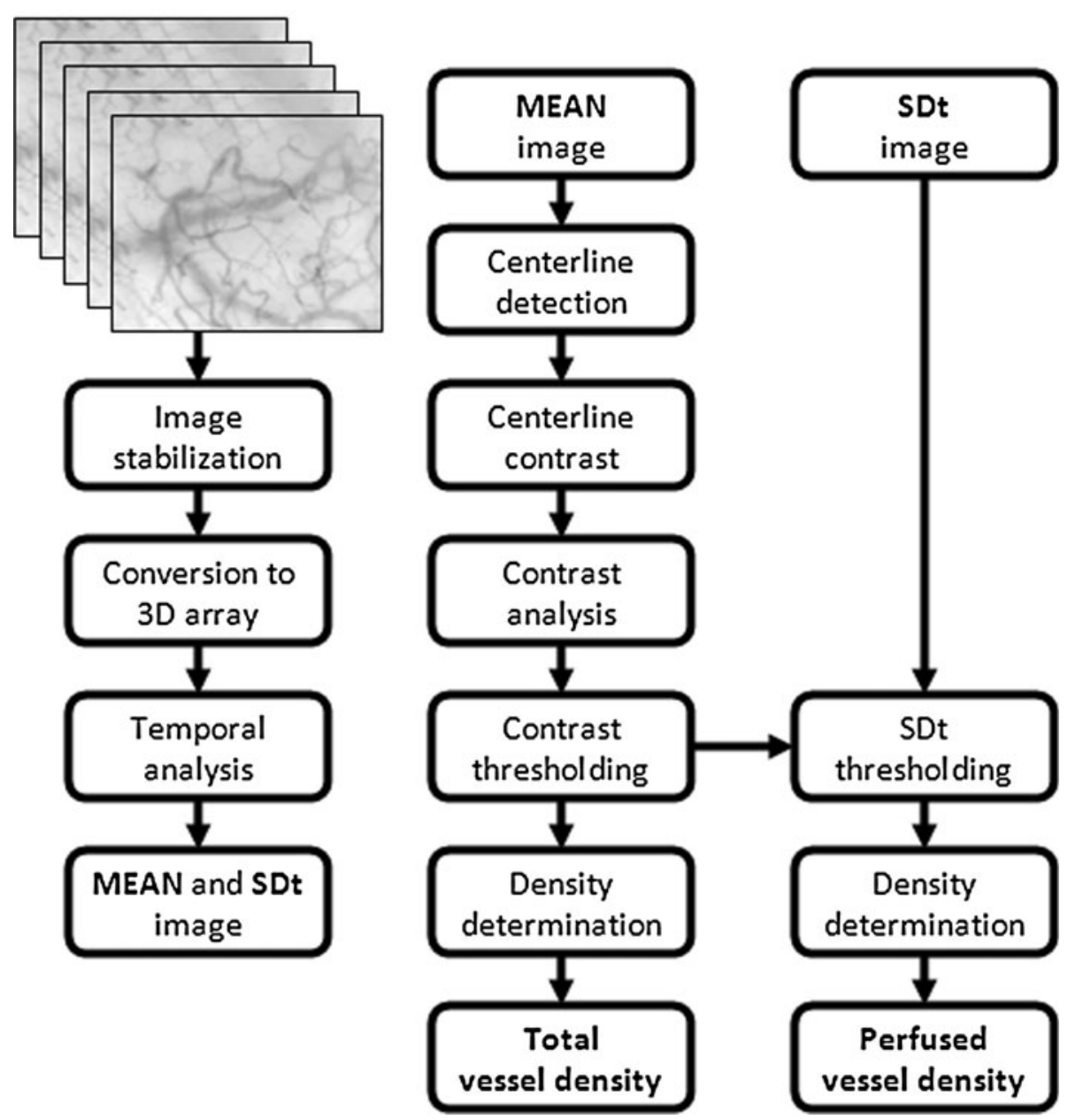

Fig. 1 A diagram schematically depicting the analysis steps required for microvascular density and perfusion assessment. First an image sequence is stabilized and converted to a $3 \mathrm{D}$ array. From the 3D array, two images are generated: (1) a time-averaged (MEAN) image; and (2) an image representing the temporal pixel intensity fluctuations,

arbitrarily set to 25 pixels with respect to the first image. Second, centerline pixels were detected based on a method described by Steger [21] and grouped into line segments as described by Staal et al. [20].

In the AVA software, the vessel wall is detected at each centerline pixel and is marked by the point where the crosssectional intensity profile has its maximum steepness. The average 'maximum steepness' of all edge points, normalized to the mean background intensity $(200 \times 200$ pixels $)$, is defined as the vessel focus score [12,13]. This focus score is used to validate detected centerlines as being actual vessel segments and to exclude noise. However, this focus score thresholding fails in case of suboptimal image focus and therefore, the user is required to manually add falsely excluded vessels and delete falsely included vessels. Thus, the semi-automatic assessment of TVD using the AVA software is still a time consuming venture $(10-30 \mathrm{~min}$, quantified as the standard deviation of intensities in time (SDt). Subsequently, the MEAN image is used to detect vessel centerlines and validate the centerlines based on contrast thresholding. Then, the SDt image can be used to classify a validated centerline as being perfused or not

depending on the level of experience of the user) requiring a significant amount of user interaction. The new method for vessel validation is not based on focus score thresholding but rather on contrast score thresholding. Several methods for scoring centerline contrast have been explored and the following was selected as it yielded the best results. The contrast at each centerline pixel was calculated as the standard deviation divided by the mean of intensity values in a $25 \times 25$ pixels area centered on the centerline pixel, multiplied by 100 [19]. The average centerline pixel contrast was defined as the centerline contrast score. In this study, vessel segments with a contrast score above a fixed, pragmatically determined, threshold of 2.00 were included in the TVD assessment.

To validate the algorithm for rapid and fully automatic detection of TVD, we reanalyzed a selection of SDF video clips that were already analyzed using AVA software for a 
study published elsewhere [5] and compared the results to (semi-)manually found microvascular densities. In that study, Boerma et al. [5] demonstrated that the sublingual microcirculation in septic patients was significantly disturbed and investigated whether this could be improved by nitroglycerin administration. Since the data set of this study has a wide spread of (semi-)manually measured (i.e., using AVA software) TVD scores, this set is convenient for the validation of the fully automatic (i.e., using the new method) TVD assessment. The clips were selected according to the flow characteristics: in the first set of clips $(n=253)$, all visible microcirculation was normally perfused (PPV $=$ $100 \%)$; and in the second set of clips $(n=72)$, some of the visible microcirculation was not perfused (PPV $<100 \%$ ). Clips were only excluded if the presence of blood, saliva, air bubbles, or other factors contaminated the microcirculatory image as was done in the original study.

Furthermore, we have tested the repeatability of the automatic measurement of TVD in video clips with normal perfusion $(\mathrm{PPV}=100 \%)$ that were highly stable (i.e., instability $\leq 5$ pixels/s) over at least $1 \mathrm{~s}$ as it is important that repeatable analyses were performed with the exact same microcirculation in the field of view (if the image is shifted somewhat, some vessels may disappear and others may appear leading to different TVD scores). We have analyzed 25 video clips five times over a time span of ten frames with every analysis started five frames later than the previous (i.e., the first analysis was performed over frames $0-10$, the second over $5-15$, etc.).

Clips were not excluded based on low image focus or contrast. As the alterations in 'small vessel' density are considered most relevant under pathophysiological conditions [5, 9], we focused on vessels with a diameter $<25 \mu \mathrm{m}$ similarly as in the original study [5].

\subsection{Microvascular perfusion assessment}

The perfusion detection method we present here, tSICA, is inspired by tLASCA [2, 6-8, 24]. In tLASCA, tissue is illuminated with a highly coherent light source such as a laser or laser diode, which results in an interference pattern (or speckle pattern) on a CCD camera. Movement of scattering particles within the illuminated tissue volume, such as flowing RBCs, influence the speckle pattern in time and quantification of the consequent pixel intensity fluctuations allows assessment of tissue perfusion. Similarly in SDF images, flowing RBCs also result in pixel intensity fluctuations in time that can be quantified to assess microvascular perfusion. For tSICA, as for the tLASCA methodology, the temporal pixel intensity fluctuations consequent to RBC passage were quantified as the standard deviation of intensities (SDt) of a pixel in a number of video frames, normalized to the mean background intensity in a $250 \times 250$ pixel area. For testing the tSICA method, the number of frames was fixed at 25 .

First, we tested the performance of the tSICA method for the binary detection of perfusion in several video simulations similar to those described previously [13] and characterized the effects of cell velocity, cell density, and the SDF imaging rate on SDt. The simulation videos showed a simulated vessel containing circular blobs with a Gaussian cross-sectional intensity profile with a standard deviation of three pixels, representing RBCs. Background intensity was set to $100 \mathrm{AU}$ and the intensity of the center of the cells was set at $50 \mathrm{AU}$. Gaussian noise was added to each frame to simulate camera noise. The cells were randomly drawn within the boundaries of a virtual vessel of $10 \mu \mathrm{m}$ wide that extends to the edges of each video frame. The density of cells was set to 2 or 4 cells per $100 \mu \mathrm{m}^{2}(=10 \times 10 \mu \mathrm{m})$ and the velocity was varied from 0 to $800 \mu \mathrm{m} / \mathrm{s}$. In addition, two imaging rates were simulated: $25 \mathrm{~Hz}$, similarly to SDF imaging rate, and $100 \mathrm{~Hz}$ to investigate the SDF hardwarerelated limitations of the tSICA method. SDt [AU] was measured at the centerline of the simulated vessels.

Second, the use of tSICA was demonstrated in one high quality SDF video clip where the microcirculation was imaged in a patient undergoing on-pump cardiac surgery [15]. The SDF video clips of this study are characterized by normal microvascular density and perfusion followed by circulatory arrest, which forms a unique scenario to test the performance of the tSICA method for binary perfusion detection. One SDF movie clip was selected based on its superior (subjectively determined) image quality in terms of stability, focus, illumination, and contrast and was analyzed before and during circulatory arrest using the new method. SDt images were generated before and during circulatory arrest to visualize the feasibility of tSICA in SDF video clips. While in the video simulation clips tSICA was only used to measure the pixel intensity fluctuations (SDt) in centerline pixels, in the SDF video clips, tSICA measured the SDt in every pixel in the whole image. Furthermore, SDt histograms were generated before and during circulatory arrest to test whether tSICA is able to separate flow from no flow.

\subsection{Data analysis}

Data analysis and plotting was performed using GraphPad Prism version 5.0 for Windows (GraphPad Method, San Diego, CA, USA). For validation of the algorithm for rapid and fully automatic assessment of TVD, the automatically determined TVD scores (new method) were plotted versus the (semi-)manually obtained TVD scores (AVA software). Correlation, linear regression, and Bland-Altman [3] analyses were performed for comparison of the two methods. To test whether the tSICA method can be used for 
the binary detection of microvascular perfusion, SDt images and corresponding SDt histograms of the same microcirculatory area before and during circulatory arrest were generated and compared.

\section{Results}

\subsection{Microvascular density}

The new method for the fully automatic analysis of TVD was very rapid and analyzed SDF movie clips within $30 \mathrm{~s}$, while analysis using the AVA software took 10-15 min. Besides the large difference in analysis time, both methods showed good agreement with respect to TVD assessment (Fig. 2). For the video clips in which PPV $=100 \%$ $(n=253)$, Pearson's $r=0.91$ and $r^{2}=0.82(P<0.01)$;
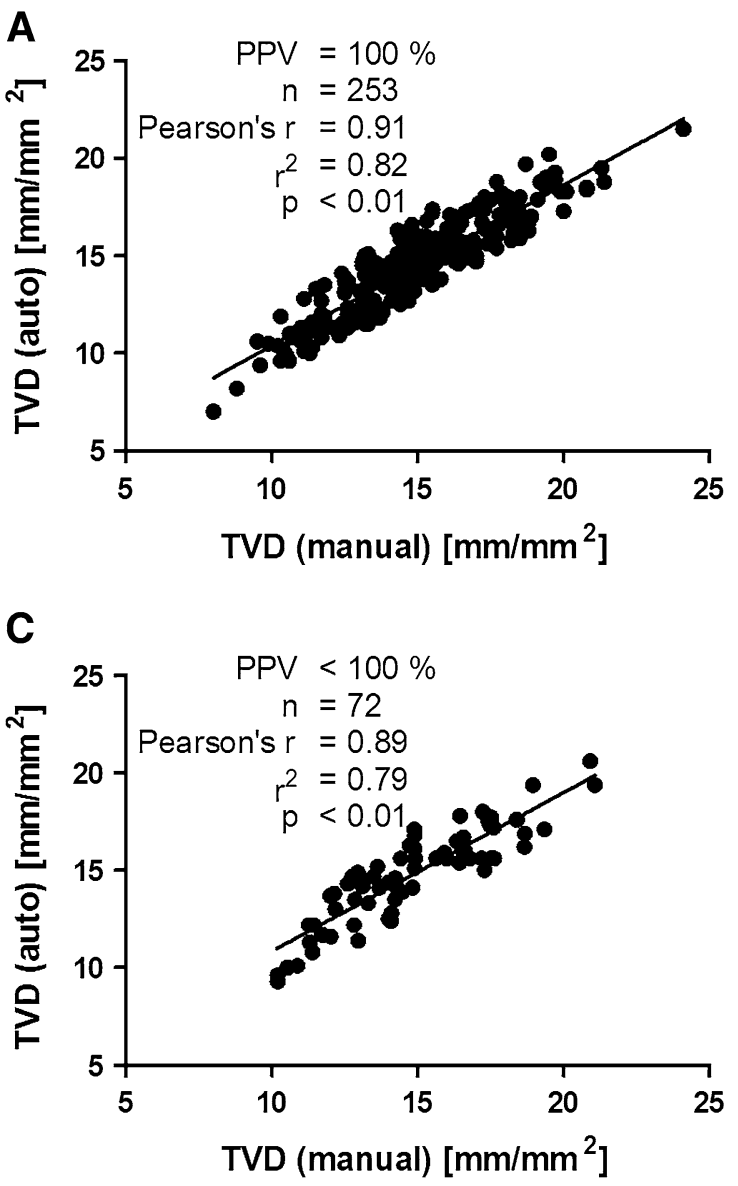

Fig. 2 Correlation, linear regression (left), and Bland-Altman (right) analysis of manually determined total vessel length (TVD) versus fully automatically determined TVD in sidestream darkfield (SDF) video clips where the proportion of perfused vessels (PPV) was $100 \%$ (upper) and less than 100\% (lower). The Bland-Altman plots show the TVD difference (i.e., the manually determined TVD minus the automatically determined TVD) versus the average TVD. For the and for the video clips in which PPV $<100 \%(n=72)$, Pearson's $r=0.89$ and $r^{2}=0.79(P<0.01)$. Furthermore, the Bland-Altman plots show an absolute bias of less than $0.5 \mathrm{~mm} / \mathrm{mm}^{2}$ in both scenarios (i.e., less than $4 \%$ of an average TVD of $14.74 \mathrm{~mm} / \mathrm{mm}^{2}$ ) (Fig. 2). For the video clips in which PPV $=100 \%$, the $95 \%$ limits of agreement were -2.68 and $1.72 \mathrm{~mm} / \mathrm{mm}^{2}$ and for the video clips in which PPV $<100 \%$, the $95 \%$ limits of agreement were -2.36 and $2.26 \mathrm{~mm} / \mathrm{mm}^{2}$. Figure 3 , in addition, depicts the repeatability of the automatic TVD assessment and shows that the average measurement standard deviation (SD; indicating the variance in analysis outcome) is $0.57 \mathrm{~mm} / \mathrm{mm}^{2}$ which is less than $4 \%$ of the average measured TVD of $14.74 \mathrm{~mm} / \mathrm{mm}^{2}$. Hence, the new method for automatic and rapid ( $<30 \mathrm{~s})$ recovery of TVD in SDF images showed good agreement for clips with normal perfusion $(\mathrm{PPV}=100 \%)$ and for clips with reduced perfusion (PPV $<100 \%)$.
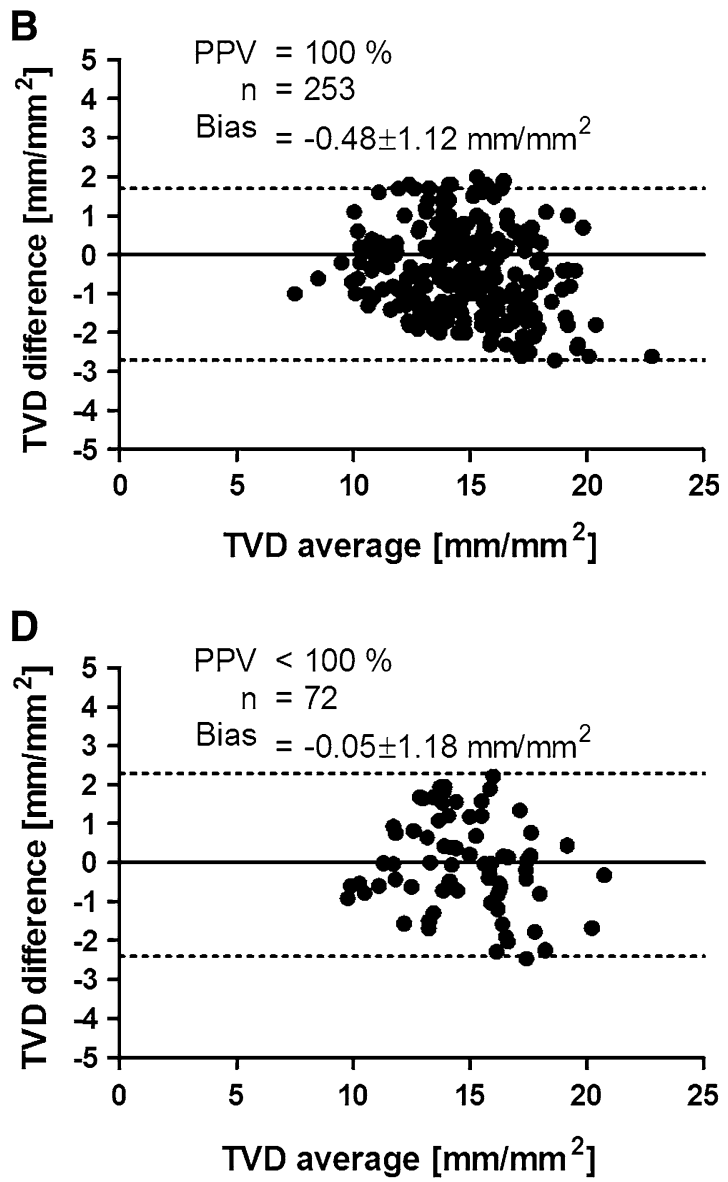

video clips in which PPV $=100 \%$, the $95 \%$ limits of agreement were -2.68 and $1.72 \mathrm{~mm} / \mathrm{mm}^{2}$ and for the video clips in which PPV $<100 \%$, the $95 \%$ limits of agreement were -2.36 and $2.26 \mathrm{~mm} / \mathrm{mm}^{2}$. The new method adequately recovers TVD and is insensitive to vessel perfusion (i.e., good agreement for clips with $\mathrm{PPV}=100 \%$ and $\mathrm{PPV}<100 \%)$ 


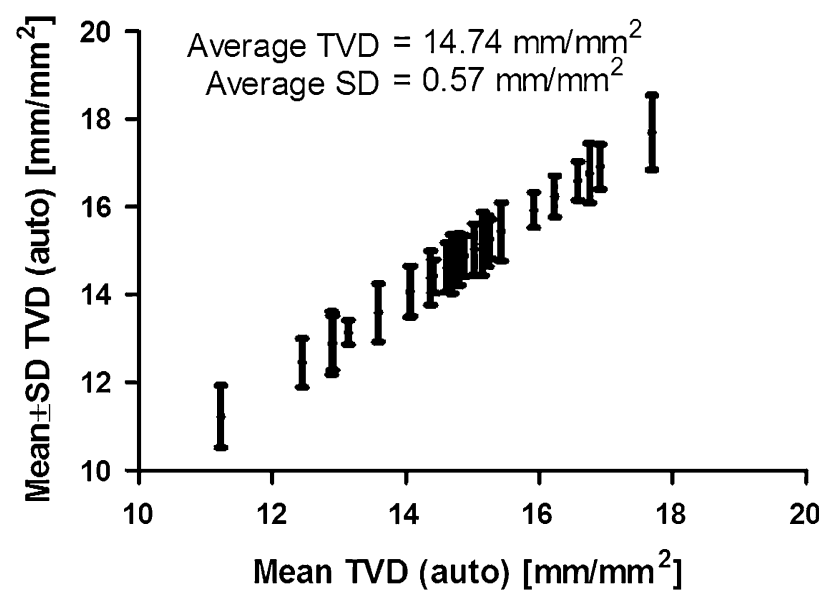

Fig. 3 The repeatability of the automatic measurement of total vessel density (TVD) in 25 video clips with normal perfusion that were highly stable (i.e., instability $\leq 5$ pixels/s) over at least $1 \mathrm{~s}$. The average measurement standard deviation (SD) is $0.57 \mathrm{~mm} / \mathrm{mm}^{2}$ which is less than $4 \%$ of the average measured TVD of $14.74 \mathrm{~mm} /$ $\mathrm{mm}^{2}$

\subsection{Microvascular perfusion}

Figure 4 shows some examples of simulated 'SDF images' with different cell densities and velocities and the corresponding SDt images for 25 and $100 \mathrm{~Hz}$ imaging rate. These examples demonstrate that high cell density in combination with high cell velocity limits the detection of variations in pixel intensities (SDt) consequent to $\mathrm{RBC}$ passages (Fig. 4, middle right image) and that this would be improved by increasing the imaging rate (Fig. 4, lower right image). Figure 5 characterizes the effects of cell density, cell velocity, and imaging rate on the ability of the tSICA method to detect perfusion. At $25 \mathrm{~Hz}$ imaging rate, a cell density of 2 cells $/ 100 \mu \mathrm{m}^{2}$ allowed perfusion detection above image noise baseline up to moderate cell velocities and a density of 4 cells $/ 100 \mu \mathrm{m}^{2}$ only allowed the detection of perfusion at very low velocities. Increasing the imaging rate to $100 \mathrm{~Hz}$ significantly improved the ability of the tSICA method to detect perfusion, but at high cell densities, this was still limited. Figure 5 furthermore shows that the SDt is low at zero flow, highest at low flow, and then decreases with increasing flow.

As shown with the video simulations, the detection of flow using the tSICA method is limited by high cell densities and velocities at $25 \mathrm{~Hz}$ imaging rate, which severely limits the applicability of this method in real SDF images. Further factors limiting the tSICA method are suboptimal image focus and contrast, reducing the pixel intensity fluctuations consequent to RBC passage (not shown). However, in some high quality SDF images with moderate cell densities and velocities, the tSICA may be applied for perfusion detection. To illustrate this, tSICA was used in one SDF video clip where the microcirculation was imaged before and during circulatory arrest [15]. SDt images (Fig. 6) and corresponding SDt histograms (Fig. 7) were generated of the same microcirculatory area at normal and stopped flow. Figure 6 shows that indeed the SDt image at normal flow is clearly brighter (indicating flow) than the SDt image at stopped flow. Furthermore, it can be seen that one vessel in the SDt image is brighter during than before circulatory arrest, which indicates low (but non-zero) flow. Figure 7 depicts the SDt histograms corresponding to the SDt images before and during circulatory arrest. The area under the histograms can be divided in three parts: first, the tissue (background) which was present both with and without perfusion; second, the perfused vessels which were only detected before cardiac arrest; and third, the non-perfused vessels which were only detected during circulatory arrest. Thus, tSICA is able to discriminate between perfused and non-perfused microvasculature in high quality SDF video clips.

\section{Discussion and conclusions}

Sublingual SDF imaging is gaining a more prominent role in clinical research. However, the offline analysis of the SDF images is still a time consuming venture (10-30 min, depending on the level of experience of the user) requiring a significant amount of user interaction. In this study, we therefore aimed to develop a rapid and fully automatic method for the assessment of microvascular density and perfusion in SDF images. We improved the algorithms for microvascular density assessment previously developed by our group (AVA software) and introduced a new method for microvascular perfusion assessment, tSICA. For the validation of the new algorithm for microvascular density assessment, we reanalyzed a selection of SDF video clips of a study in intensive care patients [5] and compared the results to (semi-)manually found microvascular densities. We showed that the new method was very rapid ( $<30 \mathrm{~s})$ and adequately recovered TVD regardless of vessel perfusion (i.e., good agreement for clips in which PPV $=100 \%$ and PPV $<100 \%)$. The performance of the tSICA method for microvascular perfusion assessment was tested in several video simulations similar to those used previously [13] and in one high quality SDF video clip where the microcirculation was imaged before and during circulatory arrest in a cardiac surgery patient [15]. With the video simulations we showed that the detection of flow using the tSICA method is limited by high cell densities and velocities at $25 \mathrm{~Hz}$ imaging rate, which severely impedes the applicability of this method in real SDF images. However, in high quality SDF video clips, the tSICA method is able to discriminate between perfused and non-perfused microvasculature.

The new method for automatic TVD assessment we present here, where vessel recognition is based on contrast 

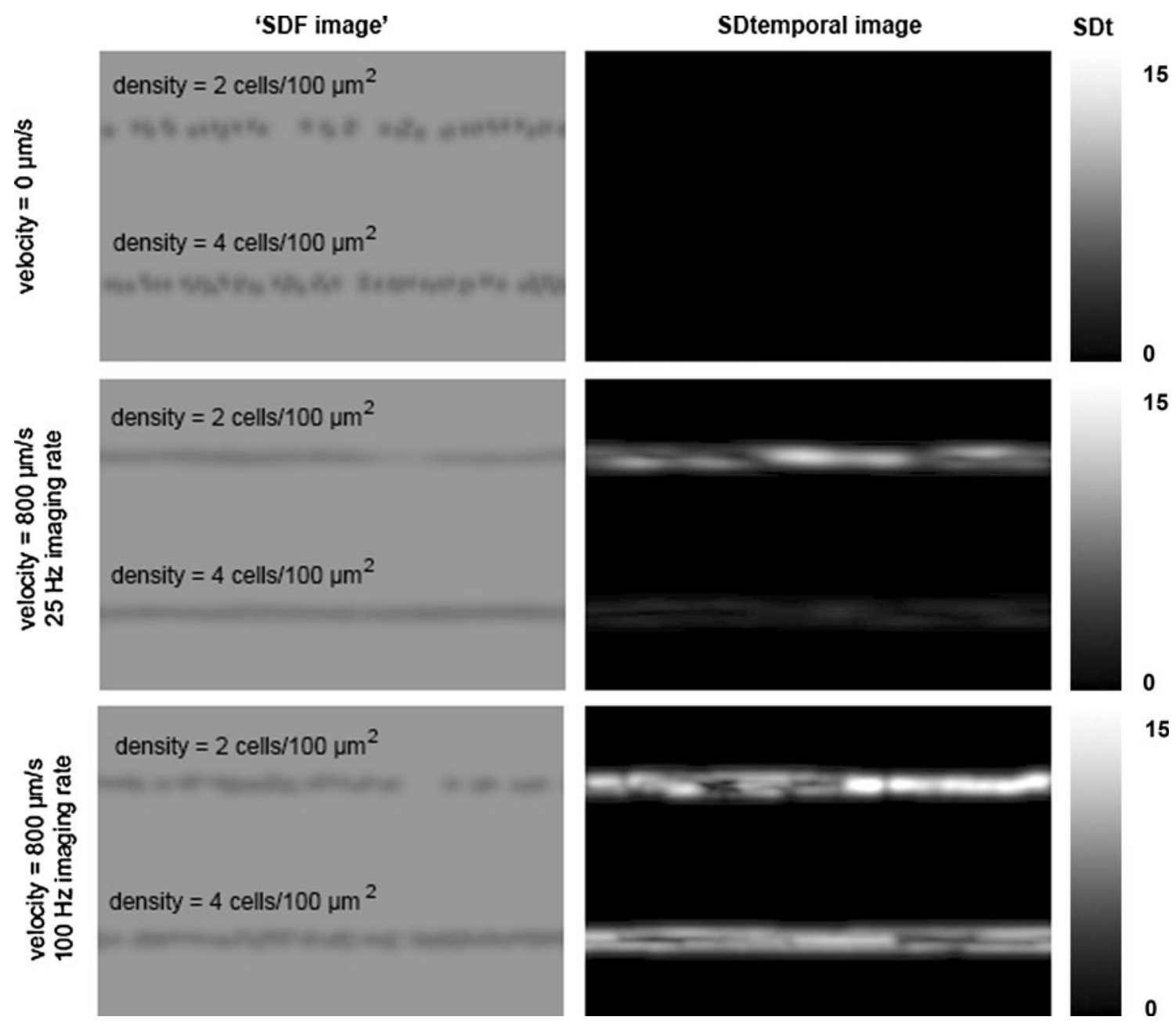

Fig. 4 Simulated vessels in 'sidestream dark field (SDF) images' (left column) and corresponding SDt images (right column) for different cell velocities ( 0 and $800 \mu \mathrm{m} / \mathrm{s}$ ), cell densities ( 2 and 4 cells $/ 100 \mu \mathrm{m}^{2}$ ) and imaging rates $(25$ and $100 \mathrm{~Hz})$. High cell density in combination

with high cell velocity limits the detection of variations in pixel intensities (SDt) consequent to RBC passages, which might be improved by increasing the imaging rate

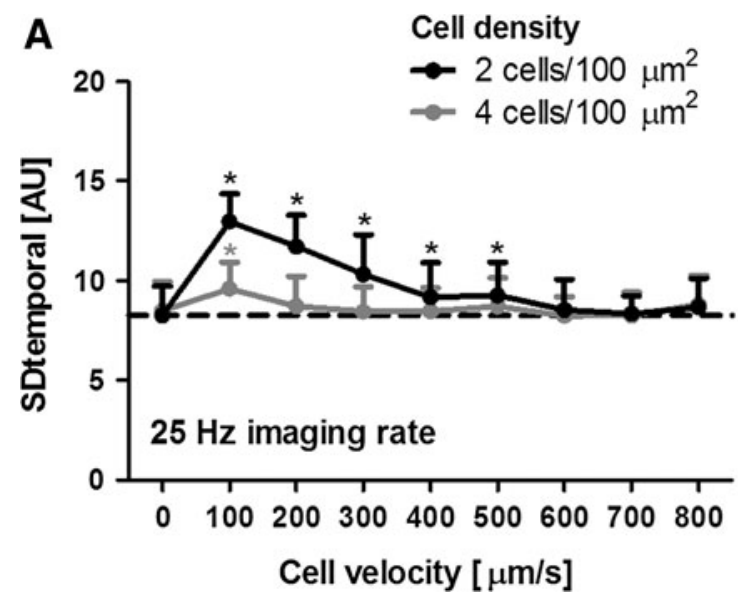

Fig. 5 Characterization of SDt as a function of cell velocity $(0-800 \mu \mathrm{m} / \mathrm{s})$, cell density ( 2 and 4 cells $\left./ 100 \mu \mathrm{m}^{2}\right)$ and imaging rate ( 25 and $100 \mathrm{~Hz} ; \mathbf{A}$ and $\mathbf{B}$, respectively). The SDt is low at zero flow,

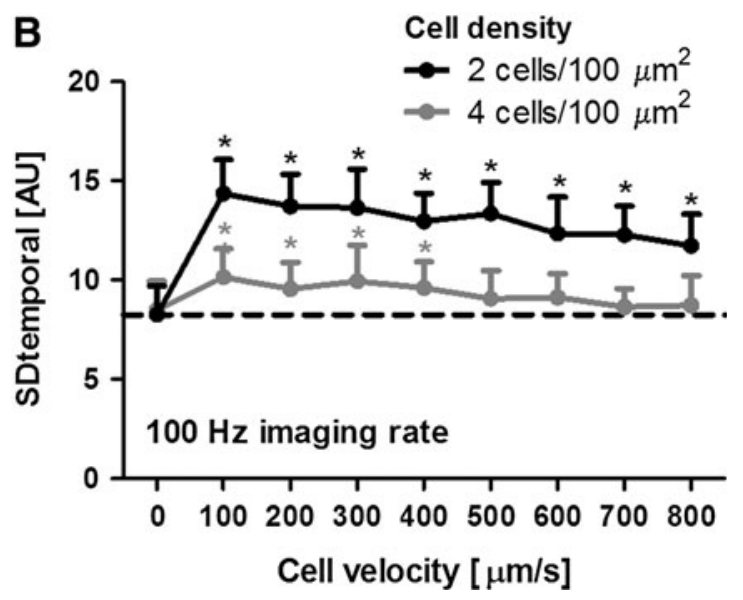

highest at low flow, and then decreases with increasing flow. $* P<0.05$ versus cell velocity $=0 \mu \mathrm{m} / \mathrm{s}$ (i.e., no perfusion) 

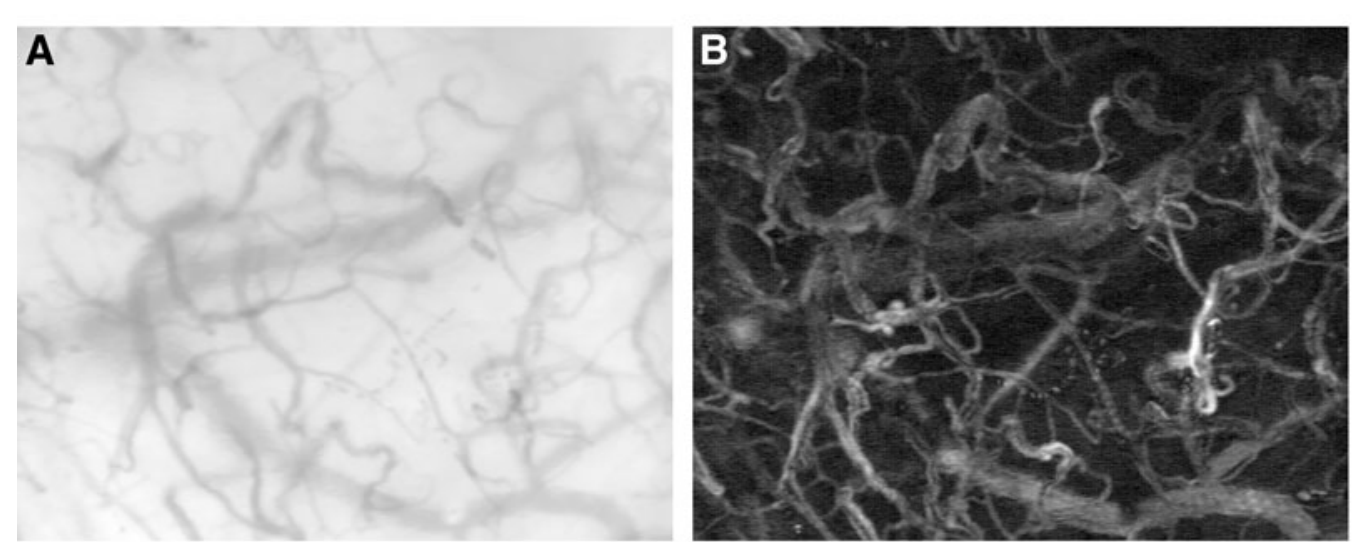

SDt
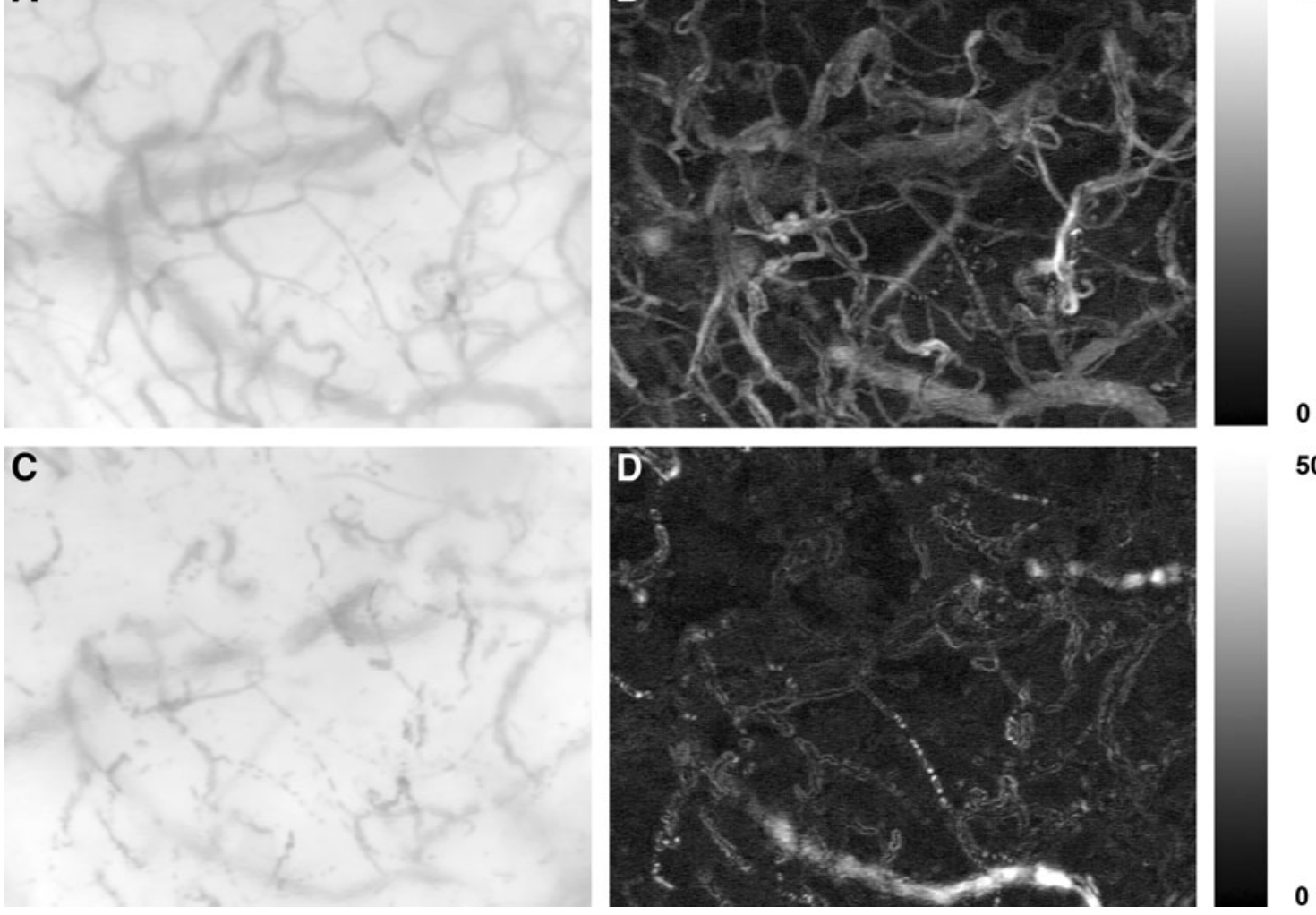

50

Fig. 6 Sidestream dark field (SDF) images before (A) and during $\mathbf{C}$ circulatory arrest and the corresponding SDt images (B and $\mathbf{D}$, respectively). The SDt image at before circulatory arrest is clearly brighter (indicating flow) than the SDt image at stopped flow

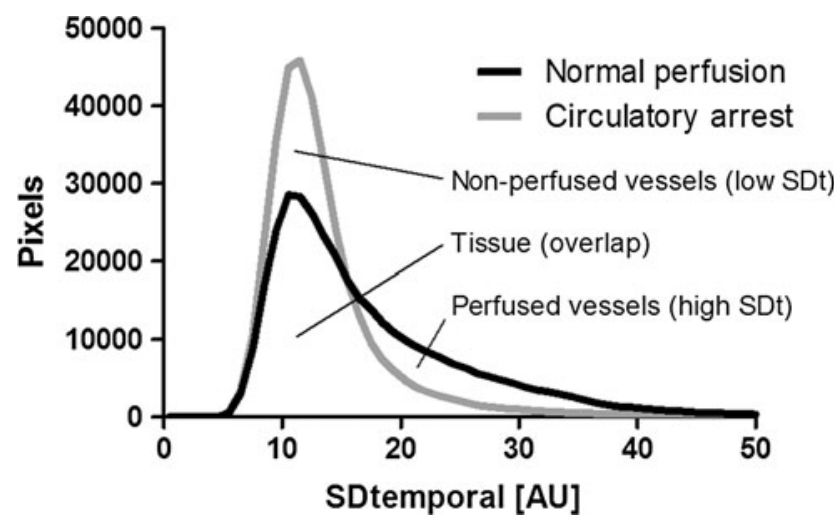

Fig. 7 SDt histograms before (black line) and during (gray line) circulatory arrest. The area under the histograms can be divided in three parts: first, the tissue (background) which was present both with and without perfusion; second, the perfused vessels which were only detected before cardiac arrest; and third, the non-perfused vessels which were only detected during circulatory arrest

rather than on focus, correlates excellently with (semi)manual TVD assessment (AVA software), with an absolute bias of less than $0.5 \mathrm{~mm} / \mathrm{mm}^{2}$ (i.e., less than $4 \%$ of an average TVD of $14.74 \mathrm{~mm} / \mathrm{mm}^{2}$ ). Furthermore, the repeatability of the automatic TVD assessment was also shown to be very good with an average measurement SD (indicating the variance in analysis outcome) of $0.57 \mathrm{~mm} / \mathrm{mm}^{2}$ (i.e., less than $4 \%$ of the average TVD). It is important to note is that the selection of clips for the validation was only based on flow characteristics and not on image focus and contrast.

According to the guidelines for microcirculatory image acquisition and analysis, 3-5 sublingual sites should be imaged per time point per patient to adequately address the heterogeneity of the microcirculation, especially during sepsis, and shock [11, 18, 23]. Using the commercially available AVA software, analyzing five sites for TVD typically takes $1 \mathrm{~h}$ (10-15 min per site), but with the new method it takes less than $5 \mathrm{~min}(\sim 1 \mathrm{~min}$ per site). In addition, as the new method is fully automatic it has perfect reproducibility. Thus, with significantly reduced analysis time, more sites can be included to more comprehensively assess the heterogeneity of the microcirculation.

In this study, we focused on measuring the density of vessels with diameter $<25 \mu \mathrm{m}$, which are considered 'small vessels' and most relevant under pathophysiological conditions according to the guidelines for microcirculatory image acquisition and analysis [11]. However, we did not consider the vessel focus score, while Dobbe et al. [13] 
have shown that low vessel focus might lead to over-estimation of the vessel diameter and thereby false exclusion from the TVD assessment. This might explain the small negative mean bias observed in the Bland-Altman [3] plots, which reflects a small underestimation of the TVD by the new method. Even so, the automatic TVD assessment still has an excellent correlation with the manual TVD assessment and is approximately 60 times faster.

With the video simulations, we showed that the detection of perfusion using the tSICA method is possible, but is limited at high cell densities and velocities at a $25 \mathrm{~Hz}$ imaging rate. A fundamental limitation of tLASCA and tSICA is that high cell velocities will result in complete blurring of the laser speckle or RBC images, respectively, and will thereby reduce the pixel intensity fluctuations to the camera noise level. In SDF images, furthermore, perfusion detection in vessels with a diameter larger than that of one RBC will be limited due to high cell densities and consequent overlapping RBCs. This, by definition, limits the quantitative measurement of RBC velocity using tSI$\mathrm{CA}$, but potentially allows the binary detection of perfusion (flow versus no flow). Other factors limiting the tSICA method are suboptimal image focus and contrast, which reduce the pixel intensity fluctuations consequent to RBC passages. This restricts the applicability of this method to real SDF images. However, with the video simulations we also showed that increasing the image rate would significantly improve the applicability of tSICA. Hence, the results obtained in the video simulations suggest that the limitations of the tSICA method are mainly hardwarerelated and could be overcome by implementing more advanced camera technology in SDF imaging (i.e., higher spatial and temporal resolution). However, whether this is actually true in real SDF images remains to be validated.

Nonetheless, we demonstrated that the tSICA method was able to discriminate between perfused and non-perfused microvasculature in one SDF video clip before and during circulatory arrest. It should be noted, however, that the scenario of normal perfusion versus circulatory arrest is quite extreme and does not reflect the range of perfusion alterations normally observed in intensive care patients. However, this scenario allowed us to present two images of the exact same microvasculature: one with and one without flow. Furthermore, it should be stressed that the background area presented in Fig. 7 is solely dependent on the thermal noise in the camera of the SDF imaging device. This is also illustrated by the non-zero SDt in the simulation videos at zero velocity. Thus, in the future, a one-time 'no-flow' measurement should be performed to characterize the camera noise and determine the perfusion discrimination level. This could be done with the same calibration unit that is provided with the SDF imaging device for calibration of the image dimensions.
In conclusion, here we present a validated method for rapid and fully automatic assessment of microvascular density. For future SDF imaging research, the automatic microvascular density assessment can be combined with manually assigning a flow score to each quadrant of the image as proposed by Spronk et al. [22], evaluated by others $[1,14]$, and included in the standard operating procedures as dictated by a consensus on microcirculatory image acquisition and analysis [11]. Although this introduces some user interaction, it allows analysis of microvascular density and perfusion in SDF video clips within a few minutes and may allow assessment of microcirculation at the bedside.

Please contact RB for collaboration for the further evaluation of rapid and fully automatic assessment of microvascular density.

\section{References}

1. Arnold RC, Parrillo JE, Phillip Dellinger R, Chansky ME, Shapiro NI, Lundy DJ, Trzeciak S, Hollenberg SM (2009) Pointof-care assessment of microvascular blood flow in critically ill patients. Intensive Care Med 35:1761-1766

2. Bezemer R, Klijn E, Khalilzada M, Lima A, Heger M, van Bommel J, Ince C (2010) Validation of near-infrared laser speckle imaging for assessing microvascular (re)perfusion. Microvasc Res 79:139-143

3. Bland JM, Altman DG (1986) Statistical methods for assessing agreement between two methods of clinical measurement. Lancet $1: 307-310$

4. Boerma EC, Mathura KR, van der Voort PH, Spronk PE, Ince C (2005) Quantifying bedside-derived imaging of microcirculatory abnormalities in septic patients: a prospective validation study. Crit Care 9:R601-R606

5. Boerma EC, Koopmans M, Konijn A, Kaiferova K, Bakker AJ, van Roon EN, Buter H, Bruins N, Egbers PH, Gerritsen RT, Koetsier PM, Kingma WP, Kuiper MA, Ince C (2010) Effects of nitroglycerin on sublingual microcirculatory blood flow in patients with severe sepsis/septic shock after a strict resuscitation protocol: a double-blind randomized placebo controlled trial. Crit Care Med 38:93-100

6. Briers JD (2001) Laser Doppler, speckle and related techniques for blood perfusion mapping and imaging. Physiol Meas 22:R35R66

7. Cheng H, Luo Q, Wang Z, Gong H, Chen S, Liang W, Zeng S (2003) Efficient characterization of regional mesenteric blood flow by use of laser speckle imaging. Appl Opt 42:5759-5764

8. Choi B, Kang NM, Nelson JS (2004) Laser speckle imaging for monitoring blood flow dynamics in the in vivo rodent dorsal skin fold model. Microvasc Res 68:143-146

9. De Backer D, Creteur J, Preiser JC, Dubois MJ, Vincent JL (2002) Microvascular blood flow is altered in patients with sepsis. Am J Respir Crit Care Med 166:98-104

10. De Backer D, Creteur J, Dubois MJ, Sakr Y, Vincent JL (2004) Microvascular alterations in patients with acute severe heart failure and cardiogenic shock. Am Heart 147:91-99

11. De Backer D, Hollenberg S, Boerma C, Goedhart P, Buchele G, Ospina-Tascon G, Dobbe I, Ince C (2007) How to evaluate the microcirculation: report of a round table conference. Crit Care 11:R101 
12. Dobbe JG, Streekstra GJ, Hardeman MR, Ince C, Grimbergen CA (2002) Measurement of the distribution of red blood cell deformability using an automated rheoscope. Cytometry 50:313-325

13. Dobbe JG, Streekstra GJ, Atasever B, van ZR, Ince C (2008) Measurement of functional microcirculatory geometry and velocity distributions using automated image analysis. Med Biol Eng Comput 46:659-670

14. Dubin A, Pozo MO, Ferrara G, Murias G, Martins E, Canullán C, Canales HS, Kanoore Edul VS, Estenssoro E, Ince C (2009) Systemic and microcirculatory responses to progressive hemorrhage. Intensive Care Med 35:556-564

15. Elbers PW, Ozdemir A, Heijmen RH, Heeren J, van Iterson M, van Dongen EP, Ince C (2010) Microvascular hemodynamics in human hypothermic circulatory arrest and selective antegrade cerebral perfusion. Crit Care Med 38:1548-1553

16. Goedhart PT, Khalilzada M, Bezemer R, Merza J, Ince C (2007) Sidestream Dark Field (SDF) imaging: a novel stroboscopic LED ring-based imaging modality for clinical assessment of the microcirculation. Opt Express 15:15101-15114

17. Groner W, Winkelman JW, Harris AG, Ince C, Bouma GJ, Messmer K, Nadeau RG (1999) Orthogonal polarization spectral imaging: a new method for study of the microcirculation. Nat Med 5:1209-1212
18. Ince C (2005) The microcirculation is the motor of sepsis. Crit Care 9:S13-S19

19. Peli E (1990) Contrast in complex images. J Opt Soc Am A 7:2032-2040

20. Staal J, Abràmoff $\mathrm{MD}$, Niemeijer $\mathrm{M}$, Viergever $\mathrm{MA}$, van Ginneken B (2004) Ridge-based vessel segmentation in color images of the retina. IEEE Trans Med Imaging 23:501-509

21. Steger C (1998) An unbiased detector of curvilinear structures. IEEE Trans Pettern Anal 20:113-125

22. Spronk PE, Ince C, Gardien MJ, Mathura KR, Oudemans-van Straaten HM, Zandstra DF (2002) Nitroglycerin in septic shock after intravascular volume resuscitation. Lancet 360:1395-1396

23. Trzeciak S, McCoy JV, Phillip DR, Arnold RC, Rizzuto M, Abate NL, Shapiro NI, Parrillo JE, Hollenberg SM (2008) Early increases in microcirculatory perfusion during protocol-directed resuscitation are associated with reduced multi-organ failure at $24 \mathrm{~h}$ in patients with sepsis. Intensive Care Med 34:2210-2217

24. Wang Z, Hughes S, Dayasundara S, Menon RS (2007) Theoretical and experimental optimization of laser speckle contrast imaging for high specificity to brain microcirculation. J Cereb Blood Flow Metab 27:258-269 